\title{
Beam Dynamics Design Using Analytical Methods for Optimizing Heat Transfer Platens Dynamics Behavior
}

\author{
Heikki Martikka ${ }^{1} \&$ Ilkka Pöllänen ${ }^{2}$ \\ ${ }^{1}$ Himtech Oy, Engineering, Ollintie 4, FIN-54100 Joutseno, Finland \\ ${ }^{2}$ SAV Oy, Savonkatu 21, FIN-45100 Kouvola, Finland \\ Correspondence: Heikki Martikka, Himtech Oy, Engineering, Ollintie 4, FIN-54100 Joutseno, Finland. Tel: \\ 358-408-393-167. E-mail: heikki.martikka@pp.inet.fi
}

Received: March 1, 2013

doi:10.5539/mer.v4n1p12

\author{
Accepted: March 15, 2013 Online Published: September 25, 2013 \\ URL: http://dx.doi.org/10.5539/mer.v4n1p12
}

\begin{abstract}
The paper presents results of using continuous beam analytical differential equation method to dynamics behavior design of complex heat transfer orthotropic platens which are used in the recovery boilers. The goal is to obtain a predictive model for increasing the reliable useful operation time. The platens are supported vertically with fixed ends. They are loaded by hot flue gas and by accumulating mass loads due to operation conditions. The loads cause alternating stresses fracturing tube branch connections. The beam deflection and bending stress histories at the junction of tube and chamber are obtained using an analytical beam model. The model gives the lowest eigenfrequency close to the measured value which is somewhat larger than that obtained by FEM model. The beam model can be used for further optimal redesign of the tube branch connections and the mean time to failure of the process equipment.
\end{abstract}

Keywords: beam theory, dynamics, fatigue design, FEM, heat transfer platens, recovery boilers

\section{Introduction}

Beams are one important basic component of the technological infrastructures. They are used extensively in static and mobile structures. Beams are mostly used as prismatic forms, solid, hollow, composite or profiles. In slender beams the transverse dynamic vibrations are excited even with moderate loads acting too close to resonances.

The object of study is heat transfer platen and other components of a typical recovery boiler.

They have three main functions. One is recycling and regeneration of cooking chemicals. The second is environmental burning of organic materials. The third is heat recovery and energy generation.

Their heat transfer platens are orthotropic and complex in geometry. They are laborious to calculate in detail even with advanced FEM (NX Nastran for FEMAP solver. Version 11.0.0. Serial number: 980-4H-3398-004F. Copyright (C2012 Siemens Product Lifecycle Management Software Inc.) as shown by Martikka, Pöllänen, and Simonen (2006). Therefore cost-effective surrogate models are needed which are much faster in calculations and accurate enough. Several surrogate models can be used to achieve this goal, like matrix, composite structure and continuous beam based methods. The aim is to construct a surrogate beam model for the platen which gives an optimal trade off between simplicity and accuracy. A general trend is to utilize surrogate models in all sciences. Generally surrogate models are combined with some optimization algorithm for maximal utility. The present beam surrogate modeling of vibrating platens is analogous with a study of optimal design of turbine discs as by Huang, Wang, Chen, and Tian (2011) using kriging surrogate models. The utility of combining surrogate models with optimization approach are discussed by Loshchilov, Schoenauer, and Sebag (2010). Jin (2011) discusses surrogate-assisted evolutionary computation combination of surrogate optimization of a swarm type structures. The collective of platens behave like a dynamic interacting swarm. These new approaches offer a promising combination of kriging surrogate models with fuzzy optimum design for future work.

Now the beam models are applied to formulate the dynamic behavior of orthotropic industrial heat transfer platens which are supported vertically with fixed ends. Their endurance is important for the whole process as discussed by Jameel, Araned, and Luks (1998). The actual aim is to maximize profit by keeping the soot layer thickness to a minimum. Beam dynamic theory is discussed by Rao (2007), Hollburg (2007), and by 
Dimarogonas and Haddad (1992). This theory is needed to build up surrogate basic models. Many recovery boilers are now required to withstand assigned seismic loads.

The optimum fuzzy design approach is discussed by Martikka and Pöllänen (2009). This fuzzy design method is based on results of Diaz (1988). This method is applicable to the present task as well. Machine design concepts are generated based on design variables ranges. The optimal one is best for the customer. At each beam there are crucial safety critical locations. Structural analysis by many researchers can be used in several calculations (Boresi, 1993; Case, Chilver, \& Ross, 1993; Ventsel \& Krauthammer, 2001). In order to perform these calculations reliable engineering design data is needed and made available by Beitz and Kuttner (Eds.) (1994). The reliability based design is important to check the reliability of this kind of extremely loaded structure as discussed by Dhillon and Singh (1981), and by Leitch (1988). One goal is to have a reliable, safe and cost-effective operation of the machines. The traditional approach does not consider the dynamics and endurance behavior of the platens. The process control is made by using measured temperatures and pressure histories to give some guidelines for activating the cleansing lance pressure and flow rates. Optimal control is important since they consume $5-12 \%$ of the superheated steam produced by the boiler. Results show that intensive cleansing may damage tubes and cause a risk of large annual loss. Subsequently, reducing the pressure by one third requires $20 \%$ increase in steam flow to maintain the same cleansing efficiency. This method is a guess and the safe optimum efficiency is not found.

In this work a simplified surrogate modeling is used. The long slender orthotropic heat transfer platens behave at the lowest bending modes as transversely vibrating beams. In this study the analytical beam models are based on differential equations approach. Literature survey of application of beam dynamics in recovery boilers showed that there are few such applications in boilers. Flow induced vibration and flutter and transverse forces can occur in the boiler between parallel platens. Unsteady flow induced forces on heat transfer tubes are discussed by Majander and Siikonen (2002). In their study the stresses and deflections are not considered.

First the internal dynamic load and masses on the platens are measured using an innovative technique.

Secondly predictive models and measurements are needed to locate acute pluggages and optimal intensity of cleansing. Analytical results are verified by using detailed model made by FEM program (NX Nastran).

The third goal is to use the beam model for further optimal redesign of the tube branch connections and the mean time to failure of the process equipment. Using the method of analytical prediction and real time measurements savings may by even $10 \%$ of the present level.

\section{Analytic Beam Dynamics Theory Is Applied Using the Classical Approach}

The objective in this section is to present an overview of the structure and design methodology and detailed geometry and loads.

\subsection{Overview of the Studied Structure}

The structure sketch is shown in Figure 1 (a). The platens are modeled as beams. The continuous beam theory is applied as discussed by Rao. The goal is to find the dynamical stress history at the critical location and to obtain satisfactory safety factors against fatigue.

\subsection{Overview of the Design Methodology}

The principle of sequential optimum fuzzy design is illustrated in Figure 1 (b). This approach can be applied in the next stage of this study (Martikka \& Pöllänen, 2009; Martikka \& Taitokari, 2011, 2012). The principle of using an overall surrogate model for the $P(s)$ function is shown in Figure 1 (c).

The structures are defined by their geometry, materials and function, loads and constraints. 


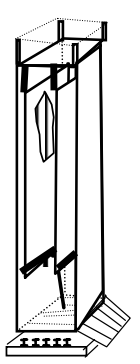

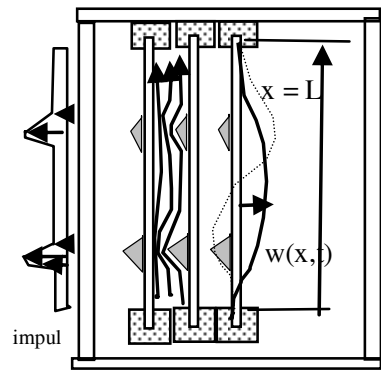

(a)

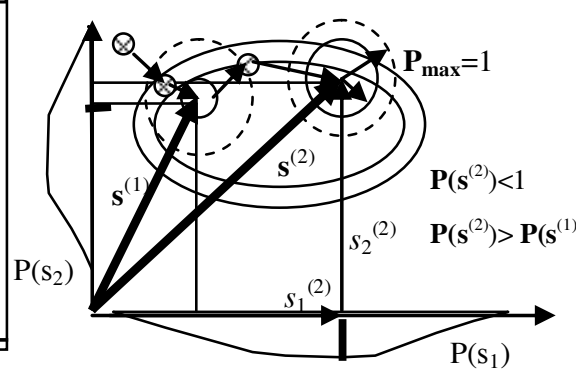

(b)

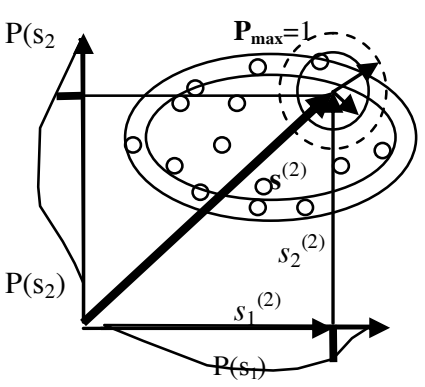

(c)

Figure 1. Recovery boiler. (a) Sketch of main structure of a recovery boiler; (b) Principle of sequential fuzzy design is shown on a two dimensional plot. The design progress is shown starting from one initial concept to final optimum. They are located with decision variable vectors. Total satisfactions are shown on the contour plot and also as a circle at tip. The maximal satisfaction is shown as a circle with unit radius; (c) Principle of using an overall surrogate model for the $P(s)$ function.

The design variables are expressed as a vector $\mathbf{x}=$ (load functions, geometry, materials). From these the decision variable vector $\mathbf{s}=($ cost, factors of safety...) $=\mathbf{s}(\mathbf{x})$ for each concept is formulated as intersection of decision variables $s_{\mathrm{k}}$

$$
s^{c}=\left[s_{1} \cap s_{2} \cap s_{3} \cap s_{4} \cap s_{5} \cap s_{6}\right]^{\text {concept } c}
$$

The design goal is maximization of the total satisfaction of the customer on the product (Diaz, 1988).

$$
P(s) \Rightarrow P(s)=P\left(s_{1}\right) \cdot P\left(s_{2}\right) \cdot \ldots . . P\left(s_{\mathrm{n}}\right), Q=\max P
$$

In this fuzzy approach the final optimum is found by exhaustive sequential search. Principle of using an overall surrogate model for the $P(s)$ function is shown in Figure 1 (c). The kriging type approach may be applied to randomly generated concepts in the design space and get the intermediate options.

\subsection{Detailed Structure of the Object of Study}

In Figure 2 some structural details are shown. Figure 2 (a) shows laboratory testing, Figure 2 (b) shows typical pluggages and Figure 2 (c) shows tube fatigue cracks.

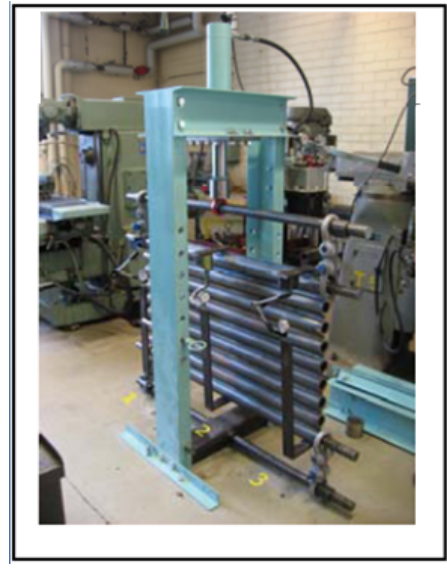

(a)

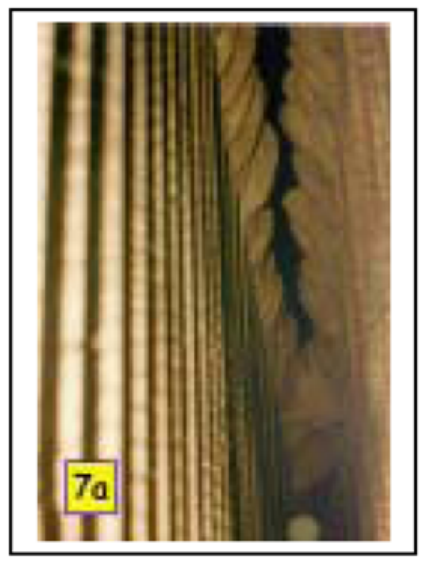

(b)

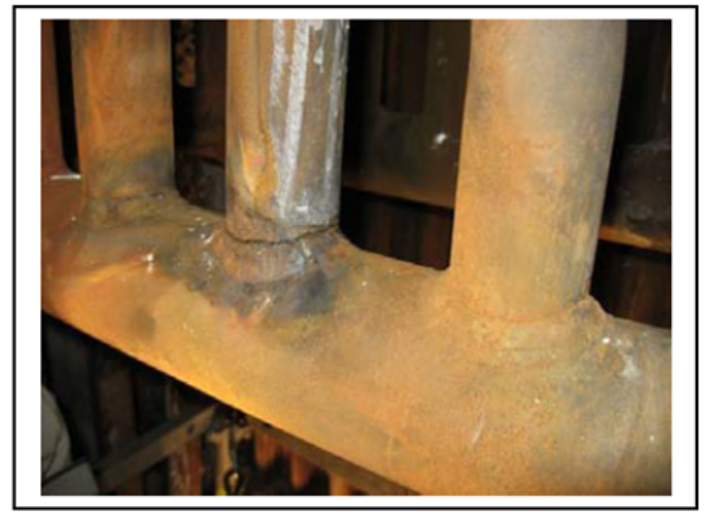

(c)

Figure 2. Orthotropic platens of the heat surfaces: (a) Laboratory testing (Talvio, 2009); (b) Typical pluggage prior to tempering by the steam; (c) Tube fatigue cracks 
This picture was taken during a chill and blow of the boiler. The pluggage is much localized and shows a nearly closed the gap between the front and back tube bundles (Jameel et al., 1998).

Geometry of the cross section of the orthotropic platen is shown in Figure 3.

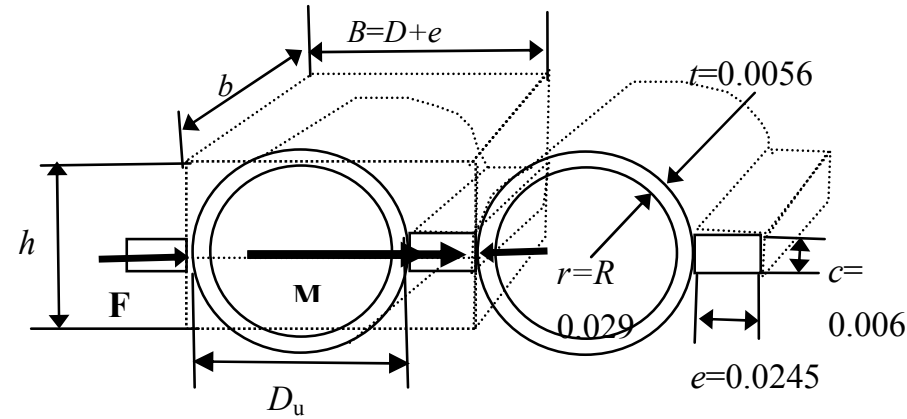

(a)

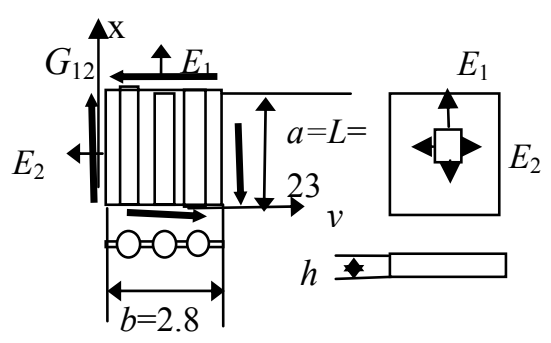

(b)

(c)

Figure 3. Orthotropic plate definitions. (a) Geometry of the cross section; (b) Orthotropic platen; (c) Surrogate plate model

The dimensions of the cross-section are

$$
D_{\mathrm{u}}=0.0635, t=0.0056, D=D_{\mathrm{u}}-t=0.0579, e=0.0245, c=0.006
$$

Here $D_{\mathrm{u}}$ is the outer diameter of the tube, $t$ is wall thickness of the tube $D$ is mean diameter and $e$ and $c$ are the length and thickness of the connection flange plate.

The bending stiffness of the cross-section of transverse length $B=D+e$ is

$$
E I=E \cdot\left[\frac{\pi}{8} D^{3} t+\frac{e c^{3}}{12}\right]
$$

Cross-section area $A_{\mathrm{b}}$ of the basic unit is sum of the tube area and the flange areas. In derivations other notations for it are used also.

\subsection{Loads on the Heat Transfer Platens}

$$
A_{\mathrm{b}}=\pi D t+e c, \quad A_{\mathrm{b}}=A(x)=A
$$

Several loads affect the heat transfer platens.

1) Flow induced vibrations are excited by the pluggages between the platens.

- Pressure variations occur depending on the channel width between two neighboring platens due to flow pressure of gas with density $\rho$ and flow velocity $v$, as $p(x, t)=\frac{1}{2} \rho v^{2}$.

- These loads cause variable bending stress under aggressive environmental load.

2) Soot blowing causes also tube fracture if it is not checked

- Consequences of tube fracture have been explosions in the factory.

Importance of soot layer control for the end user profit has been discussed by Wilhelmsen (2012) as:

- "If deposits are allowed to form on heat exchangers, the loss of efficiency can be directly related to extra fuel consumption." Simple heat transfer calculations show that a $1 \mathrm{~mm}$ deposit is approximately equivalent to a $10 \%$ efficiency loss, and a $3 \mathrm{~mm}$ deposit can reduce efficiency by up to $50 \%$.

3) Thermal and chemical loads reduce strength, wall thickness and accelerate crack growth

\section{Equation of Motion and Solution of Beam Models for the Heat Transfer Platens}

The objective in this section is to present an overview of beam dynamic theory by which the stress history results of this work are derived.

\subsection{Beam Under Distributed Transverse Forces}

Beam models are shown in Figure 4. 


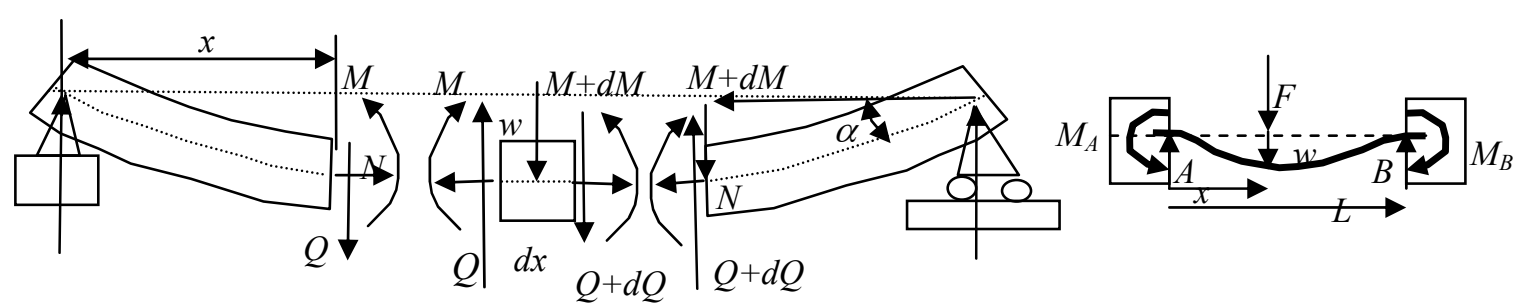

(a)

(b)

Figure 4. Beam basics: (a) Beam with free-free supports, Beam deflection $w$ and internal force resultants; (b) Beam with stiff-stiff supports and load force $F$

The linear equation of motion of the beam may be written as by Rao for forced vibrations with load $f(x, t)$

$$
\frac{\partial^{2}}{\partial x^{2}}\left[E I \frac{\partial^{2} w(x, t)}{\partial x^{2}}\right]+\rho A(x) \frac{\partial^{2} w(x, t)}{\partial t^{2}}=f(x, t)
$$

Here $w(x, t)$ is the transverse deflection of the beam at location $x$ and at time $t, \rho$ is mass density of the beam. $A$ is the cross section area of the prismatic beam, Equation (4), $E$ is the steel elastic modulus and $I$ is second moment of area and $E I$ is bending stiffness, $f(x, t)$ is the load force acting at $x$ and at time $t$.

Now the range of variation of the co-ordinate is $x=0$ to $L$ or the length of the beam.

Time variation is from an initial time $t=0$ to some seconds. The deflection at free vibrations with $f=0$ is a linear combination of all natural motions of the beam. The assumed solution is

$$
w(x, t)=\sum_{1} W_{\mathrm{i}}(x) q_{\mathrm{i}}(t)
$$

Here $W_{i}$ is the ith natural mode shape and $q_{\mathrm{i}}$ is a time dependent function.

They are determined by substituting the trial solution to the free vibration Equation (5). Here temporarily for simplicity the indices are dropped $W=W_{\mathrm{j}}$ for convenience. Now $E I$ is assumed constant. Separation of variables gives two equations

$$
\begin{gathered}
W_{x x x x}-\beta^{4} W=0 \\
\ddot{q}+\omega^{2} q=0
\end{gathered}
$$

The deflection of the beam at location $x$ and at time $t$ by Rao is

$$
w(x, t)=\sum_{1}^{\infty} w_{\mathrm{n}}(x, t)=\sum_{1}^{\infty} W_{\mathrm{n}}(x) \cdot\left(A_{\mathrm{n}} \cos \omega_{\mathrm{n}} t+B_{\mathrm{n}} \sin \omega_{\mathrm{n}} t\right)
$$

Here the eigenfrequency of eigenmode $n$ is

$$
\omega_{\mathrm{n}}=\left(\beta_{\mathrm{n}} L\right)^{2} \sqrt{\frac{E I}{\rho A L^{4}}}, \quad \beta_{n}^{4}=\frac{\rho A \omega_{n}^{2}}{E I}
$$

In order to simplify notations one may set for short $\beta_{\mathrm{n}}=\beta, \omega_{\mathrm{n}}=\omega$.

The four integration constants are determined with four boundary conditions

$$
\begin{gathered}
W(x)=C_{1}(\cos \beta x+\cosh \beta x)+C_{2}(\cos \beta x-\cosh \beta x)+ \\
+C_{3}(\sin \beta x+\sinh \beta x)+C_{4}(\sin \beta x-\sinh \beta x)
\end{gathered}
$$

\subsection{Beam Stiffly Supported at Both Ends}

Boundary conditions for the beam deflection are satisfied by the shape function $W$. The left and right ends of the beam are stiffly supported as illustrated in Figure 4 (b) with zero displacements and zero slopes. 


$$
\begin{array}{ll}
W(0)=0, & W^{\prime}(0)=0 \\
W(L)=0, & W^{\prime}(L)=0
\end{array}
$$

The first two conditions give $C_{1}=C_{3}=0$. The second two conditions give two homogeneous equations from which the ratio of the two constants can be obtained.

$$
\left[\begin{array}{cc}
\cos \beta L-\cosh \beta L & \sin \beta L-\sinh \beta L \\
-(\sin \beta L+\sinh \beta L) & \cos \beta L-\cosh \beta L
\end{array}\right]\left[\begin{array}{l}
C_{2} \\
C_{4}
\end{array}\right]=0
$$

Some lowest roots are obtained as giving the lowest eigenfrequencies

$$
\begin{gathered}
\cos \beta L \cdot \cosh \beta L=1 \\
\beta_{1} L=4.73004, \beta_{2} L=7.853, \beta_{3} L=10.99,
\end{gathered}
$$

Thus

$$
\omega_{\mathrm{n}}=\left(\beta_{\mathrm{n}} L\right)^{2} \sqrt{\frac{E I}{\rho A L^{4}}}=\left(4.73^{2}, 7.853^{2} \ldots\right) \sqrt{\frac{E I}{\rho A L^{4}}}
$$

The two constants are interdependent. The one independent constant is determined by imposing the conditions of othonormality.

$$
C_{4}=-K C_{2}, K=\frac{\cos \beta L-\cosh \beta L}{\sin \beta L-\sinh \beta L}, C_{2}=C(m, n)
$$

\subsection{Orthogonality and Orthonormality of Beam Shape Functions}

In the general Equation (5) $E I$ was variable. Now the goal is to obtain the modal modes and eigenfrequencies by dynamic equations. The following approach is used by Rao. He substitutes first a harmonic modal time function in Equation (5) and also sets $f=0$ to get free modal vibrations. Now in order to consider separate modes the indices need to be added. The result is that the equation for the shape functions for each mode $i W_{\mathrm{i}}(x)$ is obtained as

$$
\frac{\partial^{2}}{\partial x^{2}}\left[E I \frac{\partial^{2} W_{\mathrm{i}}(x)}{\partial x^{2}}\right]=\rho A(x) \omega_{\mathrm{i}}^{2} W_{\mathrm{i}}(x)
$$

Then following Rao it may be substituted back to Equation (5). Next nonzero load is assumed to act. It does not affect the modal vibrations. The following equation is obtained with nonzero load

$$
\rho A(x) \omega_{\mathrm{i}}^{2} W_{\mathrm{i}}(x) \cdot q_{\mathrm{i}}(t)+\rho A(x) W_{\mathrm{i}}(x) \frac{\partial^{2} q_{\mathrm{i}}(t)}{\partial t^{2}}=f(x, t)
$$

This modal equation may be solved using the orthogonality of the eigenfunctions

(1) First, multiplying Equation (17) by $W_{\mathrm{j}}$ on the left one obtains

$$
q_{\mathrm{i}}(t)\left[\rho A(x) \omega_{\mathrm{i}}^{2} W_{\mathrm{j}}(x) W_{\mathrm{i}}(x)\right]+\frac{\partial^{2} q_{\mathrm{i}}(t)}{\partial t^{2}}\left[\rho A(x) W_{\mathrm{j}}(x) W_{\mathrm{i}}(x)\right]=W_{\mathrm{j}}(x) f(x, t)
$$

(2) Then integrating this over the length of the beam from $x=0$ to $x=L$ yields

$$
q_{\mathrm{i}}(t)\left[\omega_{\mathrm{i}}^{2} \cdot \int_{0}^{L} \rho A(x) W_{\mathrm{j}}(x) W_{\mathrm{i}}(x) d x\right]+\frac{\partial^{2} q_{\mathrm{i}}(t)}{\partial t^{2}}\left[\int_{0}^{L} \rho A(x) W_{\mathrm{j}}(x) W_{\mathrm{i}}(x) d x\right]=\int_{0}^{L} W_{\mathrm{j}}(x) f(x, t) d x
$$

(3) The next step is to require that the shape functions of different eigenmodes are mutually orthogonal.

$$
q_{\mathrm{i}}(t)\left[\omega_{\mathrm{i}}^{2} \cdot \delta_{\mathrm{ji}}\right]+\frac{\partial^{2} q_{\mathrm{i}}(t)}{\partial t^{2}} \cdot \delta_{\mathrm{ji}}=Q_{\mathrm{j}}
$$

(4) The normal mode shape functions are made orthonormal using the property of delta function 


$$
\begin{aligned}
\delta_{\mathrm{ji}}=\int_{0}^{L} \rho A(x) W_{\mathrm{j}}(x) W_{\mathrm{i}}(x) d x & =1, \text { if } i=j, \\
& =0, \text { if } i \neq j
\end{aligned}
$$

(5) The orthonormalization condition gives equations for the constants $C(m, n)$.

The following model is assumed for $W_{\mathrm{n}}$

$$
W_{\mathrm{n}}(x)=C(m, n) \cdot\left[\left(\cos \beta_{\mathrm{n}} x-\cosh \beta_{\mathrm{n}} x\right)-K\left(\sin \beta_{\mathrm{n}} x-\sinh \beta_{\mathrm{n}} x\right)\right]=C(m, n) \cdot H_{\mathrm{n}}(x)
$$

Thus the orthonormalization constant is obtained depending on indices $m, n$ as

$$
C(m, n)^{2} \rho A \int_{0}^{L} H_{\mathrm{n}}\left(\beta_{\mathrm{n}} x\right) H_{\mathrm{n}}\left(\beta_{\mathrm{n}} x\right) d x=1 \Rightarrow C(m, n)=\frac{1}{\left[\rho A \int_{0}^{L} H_{\mathrm{n}} H_{\mathrm{n}} d x\right]^{\frac{1}{2}}}
$$

\subsection{Solution of Beam Dynamical Deflections With Various Loads}

The objective in this section is to obtain the beam deflection as superposition of modal solutions depending on the measured load history.

\subsubsection{Modal Equations}

The equation of motion of the mode $i$ is obtained from Equation (21)

$$
\ddot{q}_{\mathrm{i}}+\omega_{\mathrm{i}}^{2} q_{\mathrm{i}}=Q_{\mathrm{i}}(t)
$$

Here the generalized force is obtained from Equation (20)

$$
Q_{\mathrm{i}}(t)=\int_{0}^{t} W_{\mathrm{i}}(x) f(x, t) d x, i=1,2 \ldots
$$

Complete solution is obtained as sum of free and forced solutions

$$
q=q_{\text {free }}+q_{\text {forced }}
$$

The forced solution can be expressed with the Duhamel's integral as

$$
q_{\mathrm{i}}(t)=A_{\mathrm{i}} \cos \omega_{\mathrm{i}} t+B_{\mathrm{i}} \sin \omega_{\mathrm{i}} t+\frac{1}{\omega_{\mathrm{i}}} \int_{0}^{t} Q_{\mathrm{i}}(\tau) \sin \omega_{\mathrm{i}}(t-\tau) d \tau
$$

Substitution of this solution to Equation (5) to the trial solution of Equation (6) gives the deflection

$$
w(x, t)=\sum_{1}^{\infty} W_{\mathrm{i}}(x)\left[A_{\mathrm{i}} \cos \omega_{\mathrm{i}} t+B_{\mathrm{i}} \sin \omega_{\mathrm{i}} t+\frac{1}{\omega_{\mathrm{i}}} \int_{0}^{t} Q_{\mathrm{i}}(\tau) \sin \omega_{\mathrm{i}}(t-\tau) d \tau\right]
$$

\subsubsection{Initial and Boundary Conditions}

Boundary conditions of stiff supports are satisfied at the ends of the beam $x=0$ and $x=L$ by the previously obtained shape function $W(x)$.

$$
\begin{gathered}
w(x, t)=\sum_{1}^{\infty} W_{\mathrm{i}}(x) q_{\mathrm{i}}\left(\omega_{i} t, A_{i}, B_{i}\right) \\
w(0, t)=\sum_{1}^{\infty} W_{\mathrm{i}}(0) q_{\mathrm{i}}\left(\omega_{i} t, A_{i}, B_{i}\right)=0, \quad w^{\prime}(0, t)=\sum_{1}^{\infty} W_{\mathrm{i}}^{\prime}(0) q_{\mathrm{i}}\left(\omega_{i} t, A_{i}, B_{i}\right)=0 \\
w(L, t)=\sum_{1}^{\infty} W_{\mathrm{i}}(L) q_{\mathrm{i}}\left(\omega_{i} t, A_{i}, B_{i}\right)=0, \quad w^{\prime}(L, t)=\sum_{1}^{\infty} W_{\mathrm{i}}^{\prime}(L) q_{\mathrm{i}}\left(\omega_{i} t, A_{i}, B_{i}\right)=0
\end{gathered}
$$

Initial time conditions can be given for a mode $i$ as initial shape and initial velocity at time $t=0$ 


$$
\begin{aligned}
& w(x, 0)=\sum_{1}^{\infty} W_{\mathrm{i}}(x) q_{\mathrm{i}}\left(\omega_{i} 0, A_{i}, B_{i}\right)=w_{0}(x) \\
& \dot{w}(x, 0)=\sum_{1}^{\infty} W_{\mathrm{i}}(x) q_{\mathrm{i}}\left(\omega_{i} 0, A_{i}, B_{i}\right)=\dot{w}_{0}(x)
\end{aligned}
$$

From these two equations for each mode $i$ the two mode constants can be solved. Now all initial conditions are set to zero and the solution of free vibration is zero by setting $A_{\mathrm{i}}=0$ and $B_{\mathrm{i}}=0$

\subsubsection{Concentrated Harmonic Forces Acting on the Beam}

Using this model many load forces $F_{\mathrm{k}}, \mathrm{k}=1,2 \cdots$ may be set to act at random locations $x_{\mathrm{k}}$ and at random time $t_{\mathrm{k}}$.

When excitation starts at time $\tau=0$ one obtains for the ith time function as the forced solution only

$$
q_{\mathrm{i}}(t)=0+\frac{1}{\omega_{\mathrm{i}}} \int_{0}^{t} Q_{\mathrm{i}}(\tau) \sin \omega_{\mathrm{i}}(t-\tau) d \tau \Rightarrow q_{i, \text { forced }}
$$

Here the generalized force is by Equation (26)

$$
Q_{\mathrm{i}}(\tau)=\int_{0}^{L} W_{\mathrm{i}}(x) f(x, \tau) d x, i=1,2 \ldots
$$

Possible definitions of the load function are shown in Figure 5.

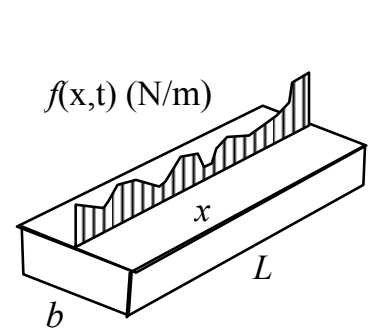

(a)

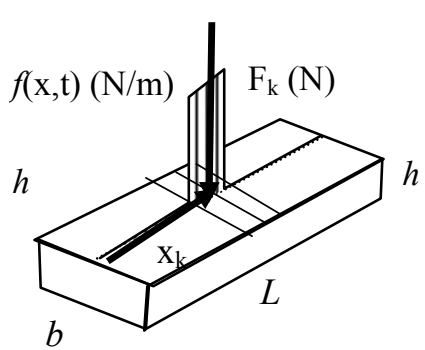

(b)

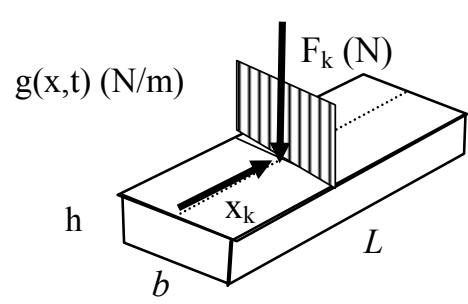

(c)

Figure 5. Definition of the load forces on beam: (a) Axial line load; (b) Axial block load; (c) Transverse line load

In Figure 5 (a) $f(x)$ is axial line load. It is a distributed load along a line upon the beam. In Figure 5 (b) an axially narrow block load model is used. The concentrated force $F_{\mathrm{k}}$ acts at location $x_{\mathrm{k}}$.

In Figure 5 (c) a transverse line load is modeled. The concentrated force acts as transverse line force

$$
f(x, t)=\frac{F_{\mathrm{k}}\left(x_{\mathrm{k}}, t\right)}{2 \Delta x}, g(x, t)=\frac{F_{\mathrm{k}}\left(x_{\mathrm{k}}, t\right)}{b}
$$

A load force with only one harmonic term is described as

$$
f(x, \tau)=F_{1} \sin \Omega_{1} \tau \cdot \delta\left(x-x_{1}\right)
$$

This force acts at location $x=x_{1}$, starts at time $t=0$ and then acts harmonically with amplitude $F_{1}$ and frequency $\Omega_{1}$.Using this option one obtains for the modal load force

$$
Q_{\mathrm{i}}(\tau)=\int_{0}^{L} W_{\mathrm{i}}(x) \cdot F_{1} \sin \Omega_{1} \tau \cdot \delta\left(x-x_{1}\right) \cdot d x, i=1,2 \ldots
$$

The property of the delta function is useful to localize the force

$$
\delta\left(x-x_{1}\right)=1, \text { if }, x=x_{1}
$$

The modal force is obtained as 


$$
Q_{\mathrm{i}}(\tau)=F_{1} \cdot W_{\mathrm{i}}\left(x_{1}\right) \cdot \sin \Omega_{1} \tau
$$

This modal force is next substituted into the equation for the time function

$$
q_{\mathrm{i}}(t)=\frac{1}{\omega_{\mathrm{i}}} \int_{0}^{t} Q_{\mathrm{i}}(\tau) \sin \omega_{\mathrm{i}}(t-\tau) d \tau
$$

The result is a time function due to one harmonic localized force excitation on mode $i$

$$
q_{\mathrm{i}}(t)=\frac{F_{1}}{\omega_{\mathrm{i}}} W_{\mathrm{i}}\left(x_{1}\right) \int_{0}^{t} \sin \Omega_{1} \tau \cdot \sin \omega_{\mathrm{i}}(t-\tau) d \tau
$$

Substituting this forced solution to Equation (29) and $A_{\mathrm{i}}=0, B_{\mathrm{i}}=0$ gives as by Rao for the beam deflection

$$
w(x, t)=\sum_{1}^{\infty} W_{\mathrm{i}}(x)\left[A_{\mathrm{i}} \cos \omega_{\mathrm{i}} t+B_{\mathrm{i}} \sin \omega_{\mathrm{i}} t+\frac{F_{1}}{\omega_{\mathrm{i}}} W_{\mathrm{i}}\left(x_{1}\right) \int_{0}^{t} \sin \Omega_{1} \tau \cdot \sin \omega_{\mathrm{i}}(t-\tau) d \tau\right]
$$

\subsubsection{Deflection Response of Beam to Two Harmonic Forces at Different Locations}

Now the start times of the load forces are set to zero. The solution is by Rao. This is used for bending stress calculations.

$$
\begin{gathered}
w(x, t)=\sum_{n=1} W_{n}(x) \cdot \frac{F_{k} W_{n}\left(x_{k}\right)}{\omega_{n}{ }^{2}-\Omega_{n}{ }^{2}}\left(\sin \Omega_{n} t-\frac{\Omega_{k}}{\omega_{n}} \sin \omega_{n} t\right) \\
w(x, t)=\sum_{n=1} W_{n}(x) \cdot q_{\mathrm{n}}(t)
\end{gathered}
$$

\subsubsection{Impact Force on the Beam}

\section{One block force}

This method is illustrated by using first one block force. The narrow time range of the activation of the force is expressed using a block function $B$ such that $B=1$ within time range $t_{\mathrm{a}}<t<t_{\mathrm{b}}$, and 0 outside. Thus

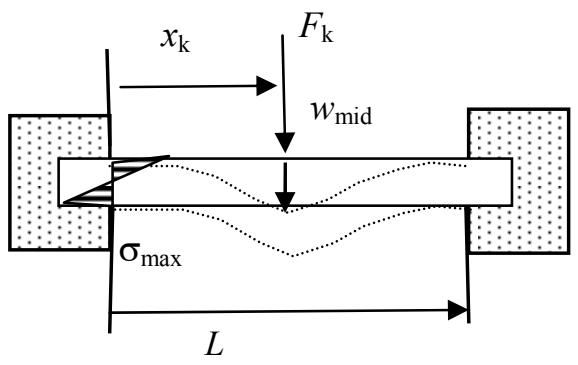

(a)

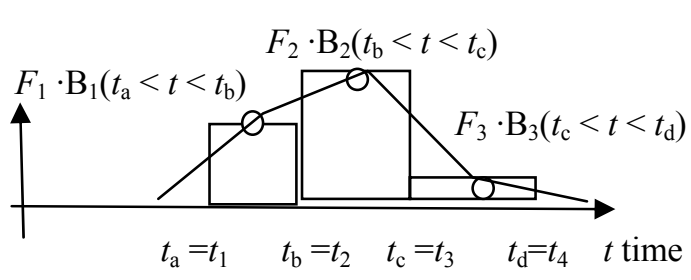

(b)

Figure 6. Impact force on beam: (a) Co-ordinate space; (b) Time space

$$
f(x, \tau)=F_{1} \sin \Omega_{1}(\tau) \cdot B\left(t_{a}, t_{b}\right) \cdot \delta\left(x-x_{1}\right), \quad \delta\left(x-x_{1}\right)=1, \text { if }, x=x_{1}
$$

Now the force starts at time 0 . Using this one obtains

$$
Q_{\mathrm{i}}(\tau)=\int_{0}^{L} W_{\mathrm{i}}(x) \cdot F_{1} \sin \Omega_{1}(\tau) \cdot B\left(t_{\mathrm{a}}, t_{\mathrm{b}}\right) \cdot \delta\left(x-x_{1}\right) \cdot d x, i=1,2 \ldots
$$

Thus

$$
Q_{\mathrm{i}}(\tau)=F_{1} \cdot W_{\mathrm{i}}\left(x_{1}\right) \cdot \sin \Omega_{1}(\tau) \cdot B\left(t_{\mathrm{a}}, t_{\mathrm{b}}\right)
$$

This may be substituted to the forced time function 


$$
q_{\mathrm{i}}(t)=0+\frac{1}{\omega_{\mathrm{i}}} \int_{0}^{t} Q_{\mathrm{i}}(\tau) \sin \omega_{\mathrm{i}}(t-\tau) d \tau
$$

Rearrangement gives

$$
q_{\mathrm{i}}(t)=\frac{F_{1}}{\omega_{\mathrm{i}}} W_{\mathrm{i}}\left(x_{1}\right) \int_{0}^{t} \sin \Omega_{1}(\tau) \cdot B\left(t_{\mathrm{a}}, t_{\mathrm{b}}\right) \cdot \sin \omega_{\mathrm{i}}(t-\tau) d \tau
$$

Now for simplicity it may be assumed that within the block there is no harmonic time dependency

$$
q_{\mathrm{i}}(t)=\frac{F_{1}}{\omega_{\mathrm{i}}} W_{\mathrm{i}}\left(x_{1}\right)\left[\int_{t=t_{a}}^{\tau=t_{b}} 1 \cdot B\left(t_{\mathrm{a}}, t_{\mathrm{b}}\right) \cdot \sin \omega_{\mathrm{i}}(t-\tau) d \tau\right]
$$

Three block forces are used to model a simple force impulse on the beam

$$
q_{\mathrm{i}}(t)=\frac{1}{\omega_{\mathrm{i}}} W_{\mathrm{i}}\left(x_{1}\right)\left[\int_{t=0}^{\tau=t} 1 \cdot \sum_{k=1}^{k=3=N k} F_{\mathrm{k}} B_{\mathrm{k}}\left(t_{\mathrm{s}}(k), t_{\mathrm{e}}(k)\right) \cdot \sin \omega_{\mathrm{i}}(t-\tau) d \tau\right]
$$

here for block $\mathrm{k}$ : $B_{\mathrm{k}} F_{\mathrm{k}}=1 \cdot F_{\mathrm{k}}$ within the interval $t_{\mathrm{s}}(\mathrm{k})<t<t_{\mathrm{e}}(\mathrm{k}), \mathrm{s}=$ start, e $=$ end

By continuing the algorithm any measured load force spectrum can be applied to the beam and the stress history can be obtained. Performing the integration gives

$$
q_{\mathrm{i}}(t)=\sum_{k=1}^{k=N k}\left\{\frac{F_{\mathrm{k}}}{\omega_{\mathrm{i}}} W_{\mathrm{i}}\left(x_{k}\right)\left[\left(-\frac{1}{\omega_{\mathrm{i}}}\right) \cos \omega_{\mathrm{i}}(t-\tau)\right]_{t_{\mathrm{s}}(k)}^{t_{\mathrm{e}}(k)}\right\}
$$

Substituting here the time limits gives

$$
q_{\mathrm{i}}(t)=-\sum_{k=1}^{k=N k}\left\{\frac{F_{\mathrm{k}}}{\omega_{\mathrm{i}}^{2}} W_{\mathrm{i}}\left(x_{\mathrm{k}}\right)\left[\cos \omega_{\mathrm{i}}\left(t-t_{\mathrm{e}}(k)\right)-\cos \omega_{\mathrm{i}}\left(t-t_{\mathrm{s}}\{k\}\right)\right]\right\}
$$

Expanding the sum gives three time functions

$$
\begin{gathered}
q_{\mathrm{i}}(t)_{F 1(x 1)}=-\frac{F_{1}}{\omega_{\mathrm{i}}^{2}} W_{\mathrm{i}}\left(x_{1}\right)\left[\cos \omega_{\mathrm{i}}\left(t-t_{2}\right)-\cos \omega_{\mathrm{i}}\left(t-t_{1}\right)\right] \\
q_{\mathrm{i}}(t)_{F 2(x 2)}=-\frac{F_{1}}{\omega_{\mathrm{i}}^{2}} W_{\mathrm{i}}\left(x_{2}\right)\left[\cos \omega_{\mathrm{i}}\left(t-t_{3}\right)-\cos \omega_{\mathrm{i}}\left(t-t_{2}\right)\right] \\
q_{\mathrm{i}}(t)_{F 3(x 3)}=-\frac{F_{3}}{\omega_{\mathrm{i}}^{2}} W_{\mathrm{i}}\left(x_{3}\right)\left[\cos \omega_{\mathrm{i}}\left(t-t_{4}\right)-\cos \omega_{\mathrm{i}}\left(t-t_{3}\right)\right]
\end{gathered}
$$

The total time function of the ith mode due to three excitation forces is a superposition

$$
q_{i}(t)_{\Sigma F}=q_{\mathrm{i}}(t)_{F 1(x 1)}+q_{i}(t)_{F 2(x 2)}+q_{i}(t)_{F 3(x 3)}
$$

The deflection response is weighted sum for each mode shape function at location $x$ and time function due to excitation forces $F_{\mathrm{k}}\left(x_{\mathrm{k}}\right)$ at locations $x_{1}, x_{2}$ and $x_{3}$

$$
w(x, t)=\sum_{1}^{\infty} W_{\mathrm{i}}(x) q_{\mathrm{i}}(t)_{\Sigma F}
$$

\section{Derivation of the Model for Stress Calculation at the Stiff Joint of the Beam}

The objective in this section is to derive the stress history modeled from the deflection model.

Industrial experience has shown the need to re-design optimally the tube joints. To reach this goal the load and stress histories are needed. Dominant stress is dynamic bending at the two lowest modes. But torsional stresses are dominant at the third mode. Now the lowest frequency is considered as most dominant. When the deflection equation is known the stresses may be calculated from the second derivative of the deflection curve 


$$
\begin{gathered}
M=E I w_{x x}, \\
\sigma=\frac{M}{I} z=\frac{M}{W}=\frac{E I w_{x x}}{I} z=E w_{x x} z=E \frac{z}{R}
\end{gathered}
$$

The maximum stress is

$$
\sigma_{\max }(x=0, t)=E \frac{1}{2} D \cdot w_{\text {xx }}(0, t)
$$

Here

$$
w_{\mathrm{xx}}(x, t)=\sum_{1}^{\infty} W_{\mathrm{n}} "(x) q_{\mathrm{n}}(t)
$$

The second derivative is needed

$$
\begin{gathered}
W_{\mathrm{k}}(x)=C \cdot\left[\left(\cos \beta_{\mathrm{k}} x-\cosh \beta_{\mathrm{k}} x\right)-K\left(\sin \beta_{\mathrm{k}} x-\sinh \beta_{\mathrm{k}} x\right)\right] \\
W_{\mathrm{k}}(x)=C \cdot H_{\mathrm{k}}(x) \\
W_{\mathrm{k}}{ }^{\prime}(x)=C \cdot H_{\mathrm{k}}{ }_{\mathrm{k}}(x)
\end{gathered}
$$

Using here the function

$$
H_{\mathrm{n}}(x)=\left(\cos \beta_{\mathrm{n}} x-\cosh \beta_{\mathrm{n}} x\right)-K\left(\sin \beta_{\mathrm{n}} x-\sinh \beta_{\mathrm{n}} x\right)
$$

One obtains the second derivative of the shape function as

$$
\begin{gathered}
W_{\mathrm{n}}{ }^{\prime}(x)=C \cdot \beta_{\mathrm{n}}{ }^{2}\left[\left(-\cos \beta_{\mathrm{n}} x-\cosh \beta_{\mathrm{n}} x\right)-K\left(-\sin \beta_{\mathrm{n}} x-\sinh \beta_{\mathrm{n}} x\right)\right] \\
W_{\mathrm{n}}{ }^{\prime}(x)=C \cdot \beta_{\mathrm{n}}{ }^{2} U\left(\beta_{\mathrm{n}} x\right)
\end{gathered}
$$

Simplification gives

$$
\begin{gathered}
W_{\mathrm{n}} "(x)=C \cdot \beta_{\mathrm{n}}{ }^{2} U\left(\beta_{\mathrm{n}} x\right), \beta_{\mathrm{n}}{ }^{2}=\omega_{\mathrm{n}} \sqrt{\frac{\rho A}{E I}} \\
W_{\mathrm{n}} "(x)=C \cdot \omega_{\mathrm{n}} \sqrt{\frac{\rho A}{E I}} \cdot U\left(\beta_{\mathrm{n}} x\right)
\end{gathered}
$$

At the stiff joint at $x=0$ the second derivative of the mode form function is

$$
\begin{gathered}
W_{\mathrm{n}}{ }^{\prime \prime}(0)=C \cdot \beta_{\mathrm{n}}^{2} U\left(\beta_{\mathrm{n}} \cdot 0\right) \\
W_{\mathrm{n}}{ }^{\prime \prime}(0)=C \cdot \beta_{\mathrm{n}}{ }^{2} \cdot\left[\left(-\cos \beta_{\mathrm{n}} 0-\cosh \beta_{\mathrm{n}} 0\right)-K\left(-\sin \beta_{\mathrm{n}} 0-\sinh \beta_{\mathrm{n}} 0\right)\right]
\end{gathered}
$$

Thus

$$
W_{\mathrm{n}}{ }^{\prime}(0)=C \cdot \beta_{\mathrm{n}}{ }^{2} \cdot(-2)
$$

Here the orthonormalization constant $C$ is given in Equation (24). Numerical solution gives $C=0.0739$. Here the eigenfrequencies are

$$
\omega_{\mathrm{n}}=\left(\beta_{\mathrm{n}} L\right)^{2} \sqrt{\frac{E I}{\rho A L^{4}}}, \quad\left(\beta_{\mathrm{n}} L\right)=4,73,7.853 \ldots \ldots
$$

The bending stress is obtained as

$$
\sigma_{\max }(x=0, t)=E \frac{1}{2} D \cdot \sum_{1}^{\infty} W_{\mathrm{n}} "(0) q_{\mathrm{n}}(t)
$$




$$
\sigma_{\max }(x=0, t)=E D \cdot \sum_{1}^{\infty}\left\{C \cdot \beta_{\mathrm{n}}{ }^{2}\right\} q_{\mathrm{n}}(t)
$$

\section{Results of Stress Calculations and Fatigue Design}

The objective in this section is to present results eigenfrequency calculations and dynamic responses and fatigue design.

\subsection{Eigenfrequencies}

In the case of the heat transfer platen of length $L=23 \mathrm{~m}$ the beam model gave the lowest eigenfrequency as

$$
\begin{aligned}
f_{1}=\frac{1}{2 \pi} \omega_{1}=4.73^{2} \sqrt{\frac{E I}{\rho A}} \frac{1}{L^{2}} & =\frac{1}{2 \pi} 4.73^{2} \sqrt{\frac{2.1 \cdot 10^{11} \cdot 4.273 \cdot 10^{-7}}{7850 \cdot 1.1656 \cdot 10^{-3}}} \frac{1}{L^{2}}=\frac{1}{2 \pi} \frac{22373}{L^{2}} \\
f_{1} & =0.67 \mathrm{~Hz}, \quad L=23 \mathrm{~m}
\end{aligned}
$$

This value $0.67 \mathrm{~Hz}$ is close to the measured frequency $f_{\text {meas }}=0.63 \mathrm{~Hz}$. Using FEM and a longer actual model $\mathrm{L}=$ $23 \mathrm{~m}$ and same cross-section the eigenfrequency is obtained as $f_{\mathrm{FEM}}=0.545 \mathrm{~Hz}$. The results agree well.

\subsection{The Dynamic Stress Response to a Dynamic Load}

Now a beam of length $L=20 \mathrm{~m}$ (not 23) is considered. One force $F_{\mathrm{k}}=100 \mathrm{~N}$ is applied at $x_{\mathrm{k}}=1 / 2 L=10 \mathrm{~m}$. The excitation frequency is set to a typical possible value of half of the resonance value

$$
f(x, t)=F_{\mathrm{k}} \sin \Omega_{\mathrm{k}} t \cdot \delta\left(x-x_{\mathrm{k}}\right), F_{\mathrm{k}}=100, \Omega_{\mathrm{k}}=0.5 \cdot \omega_{1}=0.5 \cdot 5.56 \frac{\mathrm{rad}}{\mathrm{s}}
$$

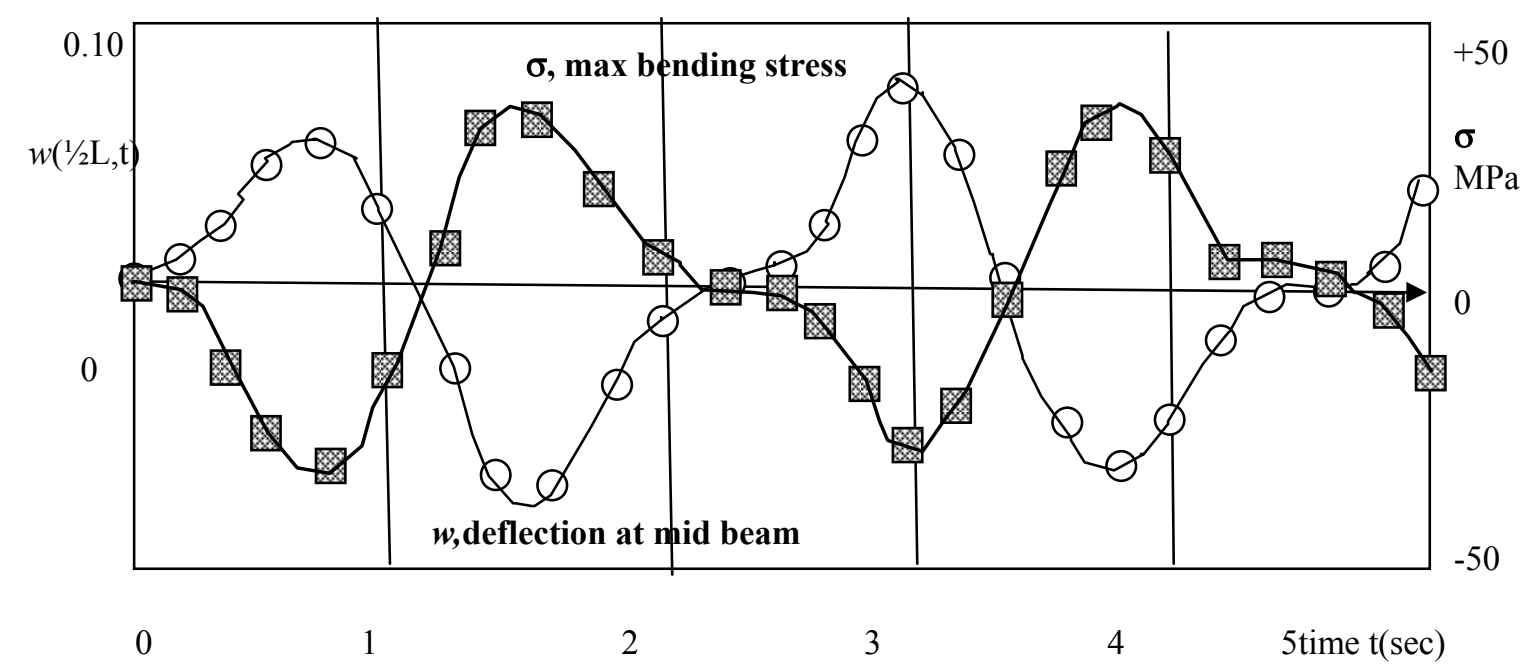

(a)

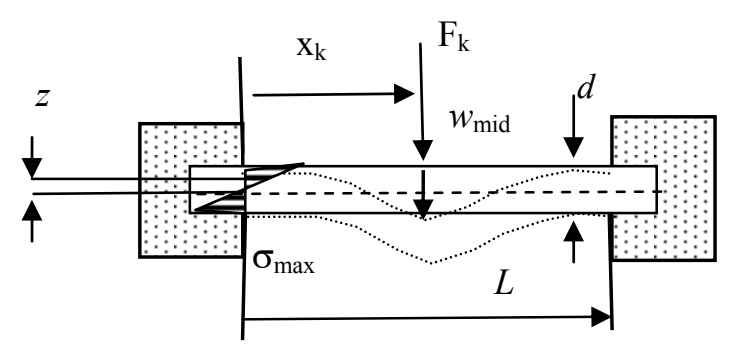

(b)

Figure 7. Results of dynamical simulations: (a) Maximum bending stress ; (b) Geometry

\subsection{Fatigue Design}

There are several methods for fatigue dimensioning. One method is to use fatigue diagrams based on assumption 
that fatigue cracks have not yet initiated (Meyer, 1985).

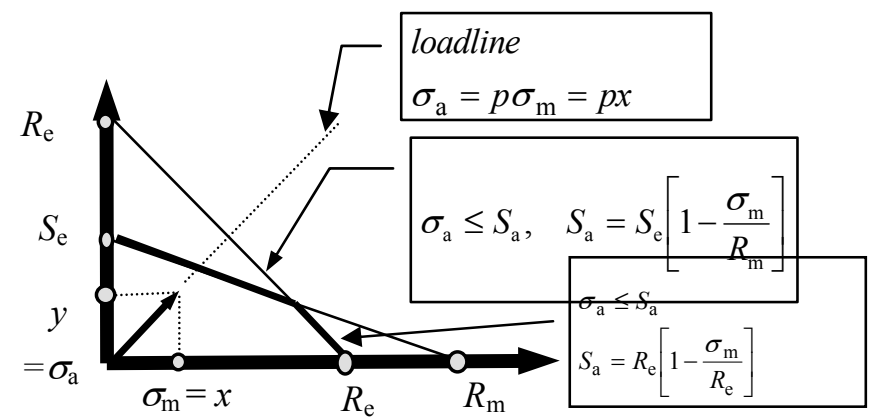

(a)

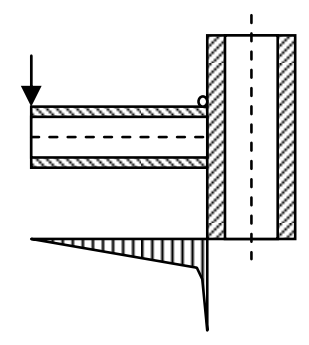

(b)

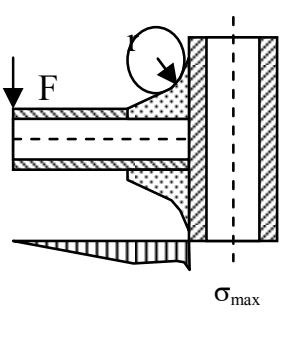

(c)

Figure 8. Fatigue modeling. (a) Fatigue diagram; (b) Conventional tube to tube joint with large stress at the joints; (c) Improved joint with small stress at the joint and even stress distribution giving even reliability

The mean stress is due to self weight of a heat transfer platen of length $L=23 \mathrm{~m}$.

$$
\sigma_{\mathrm{m}}=\rho g L
$$

The stress amplitude is the maximum bending stress

$$
\sigma_{a}=\frac{M}{I} z=\frac{E I w_{x x}}{I} z=E w_{x x} z>\sigma_{m}, \quad p=\frac{\sigma_{a}}{\sigma_{m}}>>1
$$

Thus the stress ratio $p$ is large and the bending stress is the dominant stress.

The endurance condition is

$$
R(1)=S_{\mathrm{a}}-\sigma_{\mathrm{a}} \geq 0 \rightarrow S_{\mathrm{e}}=C \cdot 0.5 R_{\mathrm{m}}=0.4 \cdot 0.5 \cdot 400=40 M P a
$$

Dynamic simulation gave the stress amplitude as $30 \mathrm{MPa}$.

$$
S_{\mathrm{a}}=\frac{1}{N}\left[\frac{p S_{\mathrm{e}}}{\frac{S_{\mathrm{e}}}{R_{\mathrm{m}}}+p}\right] \approx \frac{1}{N} S_{\mathrm{e}} \geq \sigma_{\mathrm{a}} \Rightarrow \frac{1}{N} 40>30 \Rightarrow \max \cdot N=1.33
$$

The fatigue life of tube joints can be maximized using shape optimization and protective coatings.

Figure 8 (b) shows two joints types.

- The old conventional joint type has high nominal stress and high stress concentration due to small notch radius. The fatigue lives been about a year in many cases.

- The new joint type is obtained by applying preliminary shape optimization to increase fatigue life. It has an intermediate conical tube part and large radius of curvature giving low stress concentration.

- The ratio of estimates for fatigue lives of the two models is obtained as

$$
\frac{N_{\text {new }}}{N_{\text {old }}}=\left(\frac{\sigma_{\text {new }}}{\sigma_{\text {old }}}\right)^{-3}=\left(\frac{\left\{K \sigma_{\text {nom }}\right\}_{\text {new }}}{\left\{K \sigma_{\text {nom }}\right\}_{\text {old }}}\right)^{-3}=\left(\frac{1.5 \cdot 20}{3 \cdot 40}\right)^{-3}=4^{3}=256
$$

With the new design the fatigue life at critical joint may be increased by decades.

\section{FEM Models}

Some FEM results for one heat transfer platen are shown is Figure 9. They are obtained using (NX Nastran) FEM model with 1.15 million elements. The goal was to get high accuracy in details and also in total behavior. 


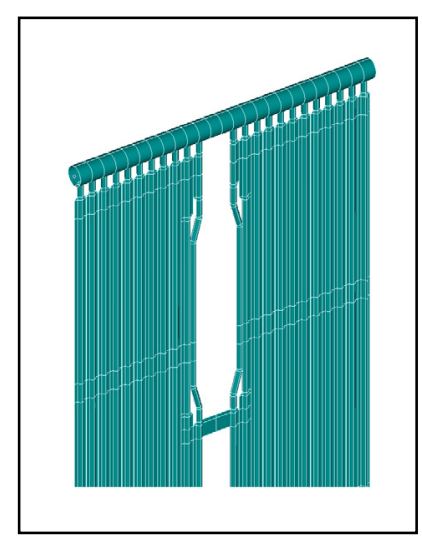

(a)

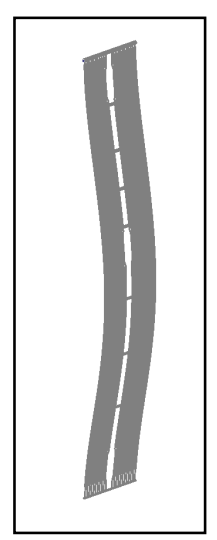

(b)

Figure 9. FEM model results: (a) Upper part. The length $L=23$ and total width $2.8 \mathrm{~m}$; (b) The first modal form with eigenfrequency $0.545 \mathrm{~Hz}$. The analytic beam model gave $0.67 \mathrm{~Hz}$ and the experimental measurement gave $0.63 \mathrm{~Hz}$

\section{Conclusions}

The main conclusions of the present study can be summarized as follows.

\section{(1) Dynamic behavior is modeled reliably with the surrogate beam model}

The continuous beam transverse dynamics model is accurate enough for describing the behavior of actual platens. Lowest bending eigenfrequencies and modes are obtained which are close to the accurate results of large FEM beam bending model. The torsional analytical dynamics is studied later. The third eigenfrequency is torsional.

\section{(2) Fatigue design is based on reliable load and beam dynamics interaction}

The beam dynamic models are useful to get reasonable dynamic bending stress history under realistic loads.

Loads on the beams are soot mass and flow induced impacts and also cleansing flow forces. By optimally targeted cleansing the annual heat transfer net profit can be increased notably with minimal maintenance and cleansing costs. The advantage of the present general dynamic beam model is that the load history can be taken into account in the fatiguing load history at the critical welds.

The large detailed FEM model gave accurate detailed stresses which are needed in detailed fatigue life analysis. These are important for optimizing the fatigue endurance of tube welds at the joints. The factor of safety against initiation of fatigue failure was obtained with a reasonably conservative fatigue diagram.

(3) Tube joint optimum design can be done using the innovative concept, fuzzy design and FEM

There are many available methods for improving notably the tube weld fatigue life or the mean time to failure. Optimal redesign by FEM of the joint geometry can be used to minimize stresses. Ceramic coating may be applied to protect the surface against corrosion and oxidation and concomitant cracks.

(4) The present approach focuses directly on the task of removing the soot with minimal damage

Nearly all conventional approaches measure process variables like temperatures, pressure and steam flow rates.

But this information is not useful for revealing the soot pluggage locations. The present method is based on measurements and their data analysis and location predictions based on dynamic behavior simulations. It can give accurate enough coordinates of soot pluggages for cleansing robots to focus on. Unnecessary cleansing and forces are avoided.

\section{Acknowledgements}

This research is financed by Himtech Oy and SAV Oy.

\section{References}

Boresi, A., Schmidt, R., \& Sidebottom, O. (1993). Advanced mechanics of materials. USA: John Wiley.

Case, J., Chilver, A. H., \& Ross, C. F. T. (1993). Strength of materials and structures with an introduction to 
finite element methods. London: Edward Arnold.

Dhillon, B. S., \& Singh, C. (1981). Engineering reliability. Wiley-Interscience.

Diaz, A. (1988). Goal aggregation in design optimization. Engineering Optimization, 13, 257-273. http://dx.doi.org/10.1080/03052158808940958

Dimarogonas, A. D., \& Haddad, S. (1992). Vibration for engineers. Prentice Hall.

Beitz, W. \& Kuttner, K. H. (Eds.). (1994). Dubbel Handbook of Mechanical Engineering. Springer Verlag.

Hollburg, U. (2007). Maschinendynamik. Oldenburg.

Jameel, M. I., Araned, H., \& Luks, G. (1998). Extending recovery boiler runtime through the temperation of steam at the soot blower. TAPPI Engineering Conference.

Jin, Y. (2011). Surrogate-assisted evolutionary computation: Recent advances and future challenges. Swarm and Evolutionary Computation, 1(2), 61-70. http://dx.doi.org/10.1016/j.swevo.2011.05.001

Leitch, R. D. (1988). Basic reliability engineering analysis. London: Butterworths.

Loshchilov, I. M., Schoenauer, \& Sebag, M. (2010). Comparison-Based Optimizers Need Comparison-Based Surrogates. Parallel Problem Solving from Nature (PPSN XI) (pp. 364-373). Springer.

Majander, P., \& Siikonen, T. (2002). A comparison of time integration methods in an unsteady low-Reynolds-number flow. International Journal for Numerical Methods in Fluids, 39, 361-390. http://dx.doi.org/10.1002/fld.286

Martikka, H., Pöllänen, I., \& Simonen, J. (2006). Design of optimally safe recovery boilers against occurrence and consequences of internal explosions (pp. 227-236). Southampton: Wessex Institute of Technology.

Martikka, H. I., \& Pöllänen, I. (2009). Multi-objective optimisation by technical laws and heuristics. Memetic computing, 1(3), 229-238. http://dx.doi.org/10.1007/s12293-009-0020-0

Martikka, H., \& Taitokari, E. (2011). Optimum Fuzzy Design of Ecological Pressurised Containers. Advances in Fuzzy Systems, 2011. http://dx.doi.org/10.1155/2011/791687

Martikka, H., \& Taitokari, E. (2012). Design of perforated shell dryings drums. Mechanical Engineering Research, 2(2), 31-44. http://dx.doi.org/10.5539/mer.v2n2p31

Meyer, J. A. (1985). Finite life under combined stress. Machine Design, August, 83-84.

Rao, S. R. (2007). Vibration of Continuous systems. USA: John Wileys.

Talvio, T. (2009). Testing of tube platens. KyAMK Osk.

Ventsel, E., \& Krauthammer, T. (2001). Thin plates and shells. New York, NY: Marcel Dekker. http://dx.doi.org/10.1201/9780203908723

Wilhelmsen. (2012). Retrieved from http://www.wilhelmsen.com/services/maritime/companies/buss/products/ MarineChemicals/Chemicals/FuelOil/Pages/571240.aspx

Huang, Z. J., Wang, C. G., Chen, J., \& Tian, H. (2011). Optimal design of aeroengine turbine disc based on $\begin{array}{lllll}\text { kriging surrogate models. Computers \& } & \text { Structures, } & 89(1-2), & \text { 27-37. }\end{array}$ http://dx.doi.org/10.1016/j.compstruc.2010.07.010

\section{Copyrights}

Copyright for this article is retained by the author(s), with first publication rights granted to the journal.

This is an open-access article distributed under the terms and conditions of the Creative Commons Attribution license (http://creativecommons.org/licenses/by/3.0/). 